\title{
Dosimetric comparison between dynamic wave arc and co-planar volumetric modulated radiotherapy for locally advanced pancreatic cancer
}

\author{
Alshaymaa Abdelghaffar \\ Sohag University \\ Noriko Kishi \\ Kyoto Daigaku \\ Ryo Ashida \\ Kyoto Daigaku \\ Yukinori Matsuo ( $\square$ ymatsuo@kuhp.kyoto-u.ac.jp ) \\ Kyoto University https://orcid.org/0000-0002-4372-8259 \\ Hideaki Hirashima \\ Kyoto Daigaku \\ Nobutaka Mukumoto \\ Kyoto Daigaku \\ Michio Yoshimura \\ Kyoto Daigaku \\ Mitsuhiro Nakamura \\ Kyoto Daigaku \\ Ahmed El Sayed Mohamed \\ Sohag University \\ Elsayed Mostafa Ali \\ Sohag University \\ Mohamed Soliman Gaber \\ Sohag University \\ Takashi Mizowaki \\ Kyoto Daigaku
}

\section{Research}

Keywords: Planning study, Locally advanced pancreatic cancer, Dynamic wave arc

Posted Date: November 10th, 2020

DOl: https://doi.org/10.21203/rs.3.rs-60459/v2 
License: (c) (i) This work is licensed under a Creative Commons Attribution 4.0 International License. Read Full License 


\section{Abstract}

Background: Dose reduction to the duodenum is important to decrease gastrointestinal toxicities in patients with locally advanced pancreatic cancer (LAPC) treated with definitive chemoradiotherapy. We aimed to compare dynamic wave arc (DWA), a volumetric-modulated beam delivery technique with simultaneous gantry/ring rotations passing the waved trajectories, with coplanar VMAT (co-VMAT) with respect to dose distributions in LAPC.

Methods: DWA and co-VMAT plans were created for 13 patients with LAPC in the pancreatic head or body. The prescribed dose was 45.6 or $48 \mathrm{~Gy}$ in 15 fractions. The dose volume indices (DVIs) for the gross tumor volume, planning target volume (PTV), stomach, duodenum, small bowel, large bowel, kidney, liver, and spinal cord were compared between the corresponding plans. The values of the gamma passing rate, monitor unit (MU), and beam-on time were also compared.

Results: The volumes of the duodenum receiving 39,42 , and 45 Gy were significantly reduced to $2.8,0.8$ and $0.15 \mathrm{~cm}^{3}$ in DWA from $3.9,1.6$ and $0.34 \mathrm{~cm}^{3}$ in co-VMAT, respectively. The mean dose of the liver and $D_{2 \mathrm{~cm} 3}$ of the planning volume for the spinal cord were significantly increased to 6.9 and $31.7 \mathrm{~Gy}$ in DWA from 5.9 and 30.2 Gy in co-VMAT, respectively. Meanwhile, there was no significant difference in the target volumes except for dose irradiated to $2 \%$ of PTV ( $110.4 \%$ in DWA vs. $109.6 \%$ in co-VMAT). There were also no significant differences in the other DVIs. Further, the gamma passing rate was $96.5 \%$ for DWA and $96.7 \%$ for co-VMAT $(p=0.65)$. The MU was significantly higher in DWA than in co-VMAT (620 vs. $589, p=0.001)$, and there was a significant increase in the beam-on time (104 sec vs. $89 \mathrm{sec}, p=$ $0.04)$.

Conclusion: DWA was superior to co-VMAT regarding dose distributions in the duodenum in LAPC, albeit with slight increasing doses to the liver and the spinal cord and increasing MU and the beam delivery time. Further evaluation is needed to know how the dose differences would affect the clinical outcomes in chemoradiotherapy for LAPC.

\section{Background}

Pancreatic cancer has poor prognosis and is one of the leading causes of cancer-related deaths worldwide [1]. Surgical resection is the standard treatment for early stage pancreatic cancer, but 30-35\% of patients are in a locally advanced stage upon diagnosis [2].These patients with locally advanced pancreatic cancer (LAPC) are commonly treated with systemic chemotherapy, but the median overall survival is only 11.9-13.6 months [3].A previous study on LAPC patients treated with first-line chemotherapy reported that $41 \%$ of the patients died without developing any distant metastases [4]. Chemoradiotherapy plays an important role in the treatment of LAPC[5].

Delivering high doses to the target organ while avoiding the organs-at-risk (OARs) is important in radiotherapy. The pancreas is surrounded by radiosensitive organs, such as the duodenum, stomach, liver, colon, and kidneys. During irradiation to the irregularly shaped target volume using three- 
dimensional conformal radiation therapy (3D-CRT), it is inevitable for these radiosensitive organs to be irradiated with high doses, thus causing gastrointestinal (GI) toxicities such as ulcer, hemorrhage, perforation, and stenosis. Conventionally fractionated 3D-CRT combined with chemotherapy has a response rate of $60 \%$, but gastroduodenal ulcers are observed in $42.3 \%$ of the patients[6,7].

Compared with 3D-CRT, high-precision radiotherapy, such as intensity-modulated radiotherapy (IMRT) and volumetric modulated radiotherapy (VMAT), enables dose escalation to the target organs with minimal increase in doses to the OARs in the treatment of LAPC. In a clinical study comparing GI toxicities between 3D-CRT and IMRT as a consequence of radiotherapy for LAPC, IMRT significantly lowered GI toxicities despite delivering higher radiation doses compared to 3D-CRT [8]. Another study comparing 3DCRT, IMRT, and VMAT for LAPC of the pancreas head showed better sparing of the OARs, especially the duodenum, small bowel, and right kidney, as well as fewer acute GI toxicities in VMAT than those in 3DCRT or IMRT [9]. Further, several reports showed that IMRT combined with chemotherapy improved overall survival (OS) and locoregional progression-free survival (LRPFS) and decreased both acute and late GI toxicities $[6,7,10]$. The dosimetric benefits of non-coplanar trajectories in minimizing the OAR doses compared to coplanar-VMAT (co-VMAT) were reported, but the non-coplanar technique led to a prolonged delivery time and increased monitor units (MUs) $[11,12]$.

A novel dose delivery method formed by simultaneous dynamic motion of the treatment couch and the linac gantry, which is called Trajectory-based Volumetric Modulated Arc Therapy (TVMAT), has been validated for use in stereotactic radiosurgery for the brain [13]. TVMAT produced comparable plans to VMAT in terms of the OAR doses while achieving better dose conformity and better OAR sparing compared to 3D-CRT plans. Another approach employing dynamic table and collimator rotations developing non-coplanar delivery technique called dynamic trajectory radiotherapy (DTRT) has been evaluated in various tumor sites including the prostate, lung, esophagus and head and neck [14]. DTRT plans could potentially spare OARs while maintaining or even improving the target coverage.

The dynamic wave arc (DWA) is a continuous non-coplanar delivery technique[15]. It allows simultaneous rotation of both radiation head unit and the 0-ring-shaped gantry, thus delivering sequential non-coplanar beams both safely and quickly without the need to rotate the couch. The clinical benefit of DWA was first reported in 15 patients with varying treatment sites, namely, breast boost, prostate, lung stereotactic body radiation therapy, and bone metastases[16]. Since then, DWA has been added to noncoplanar VMAT techniques to achieve better flexibility in dose shaping while keeping dosimetrically effective delivery. DWA has been reported to have dosimetric advantages over coplanar VMAT (co-VMAT) in tumors located in the midline of the body, such as skull base tumors and prostate cancers[17,18]. A study on the application of DWA in cases of LAPC using preclinical versions of the treatment planning system (TPS) concluded that it has comparable dosimetric distributions to co-VMAT[19]. However, there are no previous reports focused on the dosimetric advantage of DWA in pancreatic head and body cancer, which is also located in the midline of the body, using the clinically integrated version of RayStation TPS (RaySearch Laboratories, Stockholm, Sweden). Thus, this study aimed to compare DWA with co-VMAT with respect to dose distributions in locally advanced pancreatic head and body cancer, using a TPS. 


\section{Methods}

\section{Study design and patients}

This planning study evaluated 13 patients with LAPC in the pancreatic head $(n=10)$ or body $(n=3)$ who were treated with IMRT in our institution. The clinical stages (Union for International Cancer Control $7^{\text {th }}$ edition) were T3NOMO in 5 patients, T4NOMO in 7 patients, and T4N1M0 in 1 patient. The eligible criteria to this study are as follows: 1 ) primary tumors are located in the head or body of the pancreas, located close to the duodenum, 2) Planning tumor volume (PTV) sizes are less than $410 \mathrm{~cm}^{3}$ and 3) PTV is included in the field size of the Vero4DRT system (maximum, $15 \mathrm{~cm} \times 15 \mathrm{~cm}$ ).

\section{Simulation and delineation of the target organ and organs at risk}

Patients were immobilized with a vacuum pillow (BodyFIX, Medical Intelligence, Schwabmuchen, Germany) in a supine position with both arms raised above the head. Expiratory breath-hold treatmentplanning CT was performed via an intravenous contrast-enhancing agent(without oral contrast agents) using either a LightSpeed RT scanner (GE Medical Systems, Waukesha, WI, USA) with slice thickness of $2.5 \mathrm{~mm}$ or SOMATOM Definition AS (Siemens Medical Systems, Erlangen, Germany) with slice thickness of $2 \mathrm{~mm}$.

The gross tumor volume (GTV) included the tumor and metastatic lymph nodes. The clinical target volume (CTV) was defined as the GTV plus a 5-mm margin as well as the retropancreatic space and the para-aortic lymph node region between $10 \mathrm{~mm}$ superior to the celiac axis and $10 \mathrm{~mm}$ inferior to the superior mesenteric artery. The (PTV) was defined as CTV plus a 5-mm margin. The target organs and OARs were contoured based on the end-expiration phase CT. OARs were defined for the stomach, duodenum, small bowel, large bowel, kidneys, liver, and spinal cord. The planning OAR volumes (PRVs) were created with a 5-mm margin for the duodenum, small bowel, and spinal cord and a 10-mm margin for the stomach.

\section{Treatment planning}

The co-VMAT and DWA plans were created for the Vero4DRT system (Hitachi, Tokyo, Japan) using RayStation version 6.2. Dose distribution was calculated using collapsed-cone convolution algorithm with a dose grid of $2.5 \mathrm{~mm}$ (LightSpeed RT scanner) or $2.0 \mathrm{~mm}$ (SOMATOM Definition AS). The prescribed dose was 48 Gy in 15 fractions to $95 \%$ of PTV boost volume, defined as PTV excluding PRVs, while keeping the 36 Gy isodose line covering $98 \%$ of PTV volume. 
DWA treatment plans were created via synchronous rotation of the 0-ring and the gantry with optimal sequencing of dynamic multileaf collimators. A single non-coplanar trajectory called pancreas peaks up trajectory (Fig. 1) was applied to the DWA plans. The trajectory was divided into 5 segments. The 0-ring rotated from $20^{\circ}$ to $0^{\circ}, 0^{\circ}$ to $25^{\circ}, 25^{\circ}$ to $335^{\circ}, 335^{\circ}$ to $10^{\circ}$, and $10^{\circ}$ to $340^{\circ}$ during the first, second, third, fourth, and fifth segments, respectively. Simultaneously, the gantry rotated clockwise from $182^{\circ}$ to $234^{\circ}$, $234^{\circ}$ to $314^{\circ}, 314^{\circ}$ to $42^{\circ}, 42^{\circ}$ to $100^{\circ}$, and $100^{\circ}$ to $178^{\circ}$ in the five segments, respectively. The co-VMAT treatment plans consisted of one arc that rotated clockwise from $181^{\circ}$ to $179^{\circ}$.

We applied the same prescription policy and the same constraints to both the DWA and co-VMAT plans. Optimization was performed until the following criteria were met: (1) the maximum dose to the PTV was set to be $<56 \mathrm{~Gy}$, and (2) the dose to the OARs and PRVs should meet the dose constraints (Table 1) while keeping the dose coverage to PTV as mentioned above. If the PRV dose constraints cannot be fulfilled, a $5 \%$ reduction of the prescribed dose ( 45.6 Gy to $95 \%$ of PTVboost-34.2 Gy to $98 \%$ of PTV) was allowed. The dose constraints were based on the previous papers from our department $[7,20,21]$.

\section{Patient-specific quality assurance}

The patient-specific quality assurance of co-VMAT and DWA was performed using the ArcCHECK (Sun Nuclear Corp., Melbourne, FL, USA). The measured dose distribution was compared with the calculated results of treatment plans. The gamma analysis with global 3\%/3-mm gamma criteria was conducted to compare the calculated and delivered dose distributions[22]. The gamma passing rate for areas receiving isodoses above $10 \%$ was calculated using a global difference approach for the absolute dose. In addition, data on the MU and beam-on time for co-VMAT plans and DWA plans were collected.

\section{Plan evaluation and statistical analysis}

The dose volume indices (DVIs) for the following parameters were compared between co-VMAT plans and DWA plans: dose irradiated to $98 \%, 95 \%, 50 \%$, and $2 \%$ of GTV and PTV $\left(D_{98 \%}, D_{95 \%}, D_{50 \%}\right.$, and $\left.D_{2 \%}\right)$; irradiated volumes of the stomach, duodenum, large bowel, and small bowel to 39, 42, 45, and $48 \mathrm{~Gy}$ $\left(V_{39 G y}, V_{42 G y}, V_{45 G y}\right.$, and $\left.V_{48 G y}\right)$; mean dose $\left(D_{\text {mean }}\right)$ and $V_{20 G y}$ of the kidney; $D_{\text {mean }}$ of the liver; maximum dose $\left(D_{\max }\right)$ of the spinal cord; and near-maximum dose $\left(D_{2 \mathrm{~cm} 3}\right)$ of the PRV for the spinal cord. The DVIs, gamma passing rate, $\mathrm{MU}$ values, and beam-on time were compared using the paired t-test on two paired samples. All statistical analyses were performed using $\mathrm{R}$ (version 3.5.1). A $p$ value of $<0.05$ was considered statistically significant.

\section{Results}

Dose-volume indices 
The PTV ranged from $120.3 \mathrm{~cm}^{3}$ to $406.1 \mathrm{~cm}^{3}$. There was no significant difference in GTV and PTV doses except for PTV $D_{2 \%}$ between DWA and co-VMAT. PTV $D_{2 \%}$ was significantly higher in DWA plans than that in co-VMAT, but the difference was only $1 \%(111.5 \pm 2.6 \%$ vs. $110.5 \pm 2.4 \%, p<0.01)$. The details for the target volume coverage are shown in Table 2. Figure 2 shows the dose distribution in a representative case using co-VMAT and DWA. For the OARs, DWA yielded significantly lower irradiated volume of the duodenum $\left(\mathrm{V}_{39 \mathrm{~Gy}}, \mathrm{~V}_{42 \mathrm{~Gy}}\right.$, and $\left.\mathrm{V}_{45 \mathrm{~Gy}}\right)$ than co-VMAT $\left(2.8 \pm 1.9\right.$ vs. $3.9 \pm 2.3 \mathrm{~cm}^{3}$ for $\mathrm{V}_{39 \mathrm{~Gy}}, p=0.002 ; 0.8 \pm 0.6$ vs. $1.6 \pm 1.1 \mathrm{~cm}^{3}$ for $\mathrm{V}_{42 \mathrm{G} y}, p=0.004$; and $0.15 \pm 0.22$ vs. $0.34 \pm 0.30 \mathrm{~cm}^{3}$ for $\mathrm{V}_{45 \mathrm{G} y}, p=0.027$, respectively, Figure 3). Meanwhile, DWA led to a significant increase in liver $D_{\text {mean }}$, spinal cord $D_{\max }$ and $D_{2 \mathrm{~cm} 3}$ of the PRV for the spinal cord (6.9 vs. $5.9 \mathrm{~Gy}, p<0.001 ; 30.0$ vs. $29.0 \mathrm{~Gy}, p=0.041$; and 31.7 vs. $30.2 \mathrm{~Gy}, p=$ 0.011 , respectively, Table 3). There was no significant difference in DVIs of the stomach, small bowel, large bowel, or kidneys between the two plans.

Comparison of gamma passing rate, monitor unit, and beam-on time

The mean (range) 3\%/3-mm gamma passing rate was $96.5 \%$ (90.5\%-99.6\%) for DWA and $96.7 \%$ (92.3\%-100\%) for co-VMAT, with no significant difference in the gamma passing rate. Meanwhile, the MU values were significantly higher in DWA than that in co-VMAT $(620.0 \pm 74.3$ vs. $588.7 \pm 72.7, p=0.001)$. The beam-on time in DWA was significantly higher than that in co-VMAT $(104.1 \pm 24.2$ seconds vs. $88.8 \pm$ 10.9 seconds, $p=0.04)$.

\section{Discussion}

Concurrent chemoradiotherapy has been shown to increase resectability and decrease postoperative recurrence in LAPC cases[6]. Pancreatic cancer is known to have fairly low radiosensitivity[23], and thus higher doses are needed for better local control. However, this also leads to a higher frequency of $\mathrm{GI}$ toxicities. The present study showed the dosimetric benefits of DWA over co-VMAT in decreasing the intermediate and high doses to the duodenum, while preserving the PTV coverage. However, a trade-off relationship in irradiated doses among the OARs surrounding the pancreas was observed. With DWA, noncoplanar trajectories shift irradiation away from the duodenum toward other OARs namely the liver, spinal cord and left kidney. Therefore, the irradiated doses to the duodenum could be decreased with a trade-off of a slight increase in the mean liver dose and the maximum dose of the spinal cord. The other trade-off is the increase of the MUs followed by a prolongation of the treatment time.

Clinical trials assessing the benefits of escalated dose radiation with an IMRT technique in LAPC have shown significantly increased local control and OS[7,24]. Goto et al. investigated the clinical outcomes of hypofractionated IMRT in LAPC cases and reported a significantly increased LRPFS and OS in cases treated with doses $\geq 45$ Gy in 15 fractions. Krishnan et al. also reported significant improvements in LRPFS and OS in LAPC patients treated with dose-escalated IMRT (biologically effective dose [BED], >70 Gy). However, although dose-escalating IMRT trials have shown promising results, GI toxicities remain a major concern. Further reduction of GI toxicities is needed to adequately satisfy the need for dose escalation to achieve better local control. 
Previous reports have showed the association between DVIs and GI toxicities, but the prescribed doses and fractionations varied among these reports [25-28](Table 4). The BED is commonly used to compare such various doses and fractionation schedules with an alpha/beta ratio of $10 \mathrm{~Gy}$ (BED10) for acute toxicities and $3 \mathrm{~Gy}$ (BED3) for late toxicities in the duodenum. Considering the DVIs converted to BED10 and BED3, the DVIs for the duodenal toxicities proposed by the reports are consitent with most of the DWA plans in the present study (Table 4). Our data indicate that the use of DWA may be beneficial to decrease both acute and late duodenal toxicities, which is crucial for dose escalation in LAPC. However, it remains unclear whether the dosimetric superiority directly reduces $\mathrm{Gl}$ toxicities because the toxicities are caused by multiple treatment-related factors such as chemotherapy and low platelet count[29,30].

Several methods of respiratory motion management have been investigated for the delivery of radiotherapy to pancreatic cancer patients. Abdominal compression, which reduces target motion by applying pressure to the patient's abdomen limiting diaphragmatic motion, is thought to cause pain and discomfort to the patient and alter the reproducibility of the OARs which may impact treatment delivery accuracy [31]. Breath-hold techniques can be used to reduce tumor motion during radiotherapy. The motion magnitude of the pancreatic head was found to be larger during inhalation breath-holds than during exhalation breath-holds [32]. Nakamura et al. have concluded that exhalation breath-hold is tolerable[33]. In their study, all the 10 tested pancreatic cancer patients succeeded in maintaining exhalation breath-holds for 20 seconds. In the present study, the breath-hold at the end of exhalation was assumed for both co-VMAT and DWA plans. DWA needed more MUs than co-VMAT (620 vs. 589), as well as prolongation of treatment time (104.1 sec vs. $88.8 \mathrm{sec})$. The 15 -sec increase of the beam delivery time would lead to an increase in the number of the breath-holding sessions by one. The increase in MUs is presumably due to the increased beam path length because DWA irradiates a tumor from non-coplanar angles.

There are some limitations in the present study. First, the sample size is small, but it is still larger than that in a previous report on DWA that used a preclinical version of TPS [19]. Second, we focused on centrally located LAPC, and thus the benefit of DWA in tail pancreatic cancer or LAPC with larger PTV remains unknown. Given that this is a planning study, further investigation is warranted to explore the impact of increased liver and spinal cord doses on clinical outcomes, and for which cancer types and for which DWA trajectories can be optimal. Third, the present study evaluated only single-arc or singletrajectory plans for both DWA and co-VMAT. One reason for that is the shorter time for beam delivery preferable for breath-hold techniques. The other reason is that multiple coplanar arcs increase the tongueand-groove effect because the Vero4DRT system cannot rotate its collimator.

\section{Conclusions}

DWA was superior to co-VMAT regarding dose distributions in the duodenum in LAPC, albeit with slight increasing doses to the liver and the spinal cord and increasing MU and beam delivery time. Further evaluation is needed to know how the dose differences would affect clinical outcomes in 
chemoradiotherapy for LAPC. These findings would help further the clinical studies of IMRT for LAPC, with escalating doses to the tumor while keeping less duodenal toxicities.

\section{Declarations}

\section{Ethics approval and consent to participate}

This study was approved by the Institutional Review Board of Kyoto University Hospital (R1446) and was performed in accordance to the Declaration of Helsinki (1975, as revised in 2013). The need for written informed consent was waived.

\section{Consent for publication}

Not applicable.

\section{Availability of data and materials}

The datasets used and/or analyzed during the current study are available from the corresponding author on reasonable request.

\section{Competing interests}

Takashi Mizowaki has an advisory contract with Hitachi, Ltd. Other authors declare that they have no conflict of interest.

\section{Funding}

This work was supported by the Core-to-Core Program, JSPS (JPJSCCA20200009).

\section{Authors' contributions}

A.A. performed the planning study and wrote the manuscript. Y.M. supervised the study. R.A. and N.K. contributed the analysis of the results and supported designing the figures. H.H. performed the quality assurance. All the co-authors commented on the manuscript.

\section{Acknowledgements}

We would like to thank Editage (www.editage.jp) for English language editing.

\section{Abbreviations}

LAPC: Locally advanced pancreatic cancer; DWA: dynamic wave arc; VMAT: Volumetric Modulated Arc therapy; co-VMAT: coplanar VMAT; DVIs: dose volume indices; PTV: planning target volume; MU: monitor unit; OARs: organs at-risk; 3D-CRT: three-dimensional conformal radiation therapy; GI: gastrointestinal; 
IMRT: intensity-modulated radiotherapy; CT: computed tomography; OS: overall survival; LRPFS: locoregional progression-free survival; TPS: treatment planning system; GTV: gross tumor volume; CTV: clinical target volume; PRV: planning OAR volume; BED: biological effective dose.

\section{References}

1. Bray F, Ferlay J, Soerjomataram I, Siegel RL, Torre LA, Jemal A. Global cancer statistics 2018 : GLOBOCAN estimates of incidence and mortality worldwide for 36 cancers in 185 countries. CA Cancer J Clin. 2018;68:394-424.

2. Stathis A, Moore MJ. Advanced pancreatic carcinoma: current treatment and future challenges. Nat Rev Clin Oncol. 2010;7:163-72.

3. Hammel P, Huguet F, van Laethem J-L, Goldstein D, Glimelius B, Artru P, et al. Effect of Chemoradiotherapy vs Chemotherapy on Survival in Patients With Locally Advanced Pancreatic Cancer Controlled After 4 Months of Gemcitabine With or Without Erlotinib: The LAP07 Randomized Clinical Trial. JAMA. 2016;315:1844-53.

4. Peixoto RD, Speers C, McGahan CE, Renouf DJ, Schaeffer DF, Kennecke HF. Prognostic factors and sites of metastasis in unresectable locally advanced pancreatic cancer. Cancer Med. 2015;4:1171-7.

5. Robin TP, Goodman KA. Radiation therapy in the management of pancreatic adenocarcinoma: review of current evidence and future opportunities. Chinese Clin Oncol. 2017;6:28.

6. Lee KJ, Yoon HI, Chung MJ, Park JY, Bang S, Park S, et al. A Comparison of Gastrointestinal Toxicities between Intensity-Modulated Radiotherapy and Three-Dimensional Conformal Radiotherapy for Pancreatic Cancer. Gut Liver. 2016;10:303-9.

7. Goto Y, Nakamura A, Ashida R, Sakanaka K, Itasaka S, Shibuya K, et al. Clinical evaluation of intensity-modulated radiotherapy for locally advanced pancreatic cancer. Radiat Oncol. 2018;13:118.

8. Prasad S, Cambridge L, Huguet F, Chou JF, Zhang Z, Wu AJ, et al. Intensity modulated radiation therapy reduces gastrointestinal toxicity in locally advanced pancreas cancer. Pract Radiat Oncol. 2016;6:78-85.

9. Jin L, Wang R, Jiang S, Yue J, Liu T, Dou X, et al. Dosimetric and clinical toxicity comparison of critical organ preservation with three-dimensional conformal radiotherapy, intensity-modulated radiotherapy, and RapidArc for the treatment of locally advanced cancer of the pancreatic head. Curr Oncol. 2016;23:e41-8.

10. Yovino S, Poppe M, Jabbour S, David V, Garofalo M, Pandya N, et al. Intensity-modulated radiation therapy significantly improves acute gastrointestinal toxicity in pancreatic and ampullary cancers. Int J Radiat Oncol Biol Phys. 2011;79:158-62.

11. Audet C, Poffenbarger BA, Chang P, Jackson PS, LundahI RE, Ryu SI, et al. Evaluation of volumetric modulated arc therapy for cranial radiosurgery using multiple noncoplanar arcs. Med Phys. 2011;38:5863-72. 
12. Uto M, Mizowaki T, Ogura K, Hiraoka M. Non-coplanar volumetric-modulated arc therapy (VMAT) for craniopharyngiomas reduces radiation doses to the bilateral hippocampus: a planning study comparing dynamic conformal arc therapy, coplanar VMAT, and non-coplanar VMAT. Radiat Oncol. 2016;11:86.

13. Wilson B, Otto K, Gete E. A simple and robust trajectory-based stereotactic radiosurgery treatment. Med Phys. 2017;44:240-8.

14. Fix MK, Frei D, Volken W, Terribilini D, Mueller S, Elicin O, et al. Part 1: Optimization and evaluation of dynamic trajectory radiotherapy. Med Phys. 2018;45:4201-12.

15. Mizowaki T, Takayama K, Nagano K, Miyabe Y, Matsuo Y, Kaneko S, et al. Feasibility evaluation of a new irradiation technique: three-dimensional unicursal irradiation with the Vero4DRT (MHI-TM2000). J Radiat Res. 2013;54:330-6.

16. Burghelea M, Verellen D, Dhont J, Hung C, Gevaert T, Van den Begin R, et al. Treating patients with Dynamic Wave Arc: First clinical experience. Radiother Oncol. 2017;122:347-51.

17. Uto M, Mizowaki T, Ogura K, Miyabe $\mathrm{Y}$, Nakamura M, Mukumoto N, et al. Volumetric modulated Dynamic WaveArc therapy reduces the dose to the hippocampus in patients with pituitary adenomas and craniopharyngiomas. Pract Radiat Oncol. 2017;7:382-7.

18. Nakamura K, Mizowaki T, Uto M, Mukumoto N, Miyabe Y, Ono T, et al. EP-1553: Dose reduction of femoral heads using volumetric-modulated Dynamic WaveArc for prostate cancer. Radiother Oncol. 2017;123:S836-7.

19. Burghelea M, Verellen D, Poels K, Hung C, Nakamura M, Dhont J, et al. Initial characterization, dosimetric benchmark and performance validation of Dynamic Wave Arc. Radiat Oncol. Radiation Oncology; 2016;11:63.

20. Goto Y, Ashida R, Nakamura A, Itasaka S, Shibuya K, Akimoto M, et al. Clinical results of dynamic tumor tracking intensity-modulated radiotherapy with real-time monitoring for pancreatic cancers using a gimbal mounted linac. Oncotarget. 2018;9:23628-35.

21. Nakamura A, Hiraoka $M$, Itasaka $S$, Nakamura $M$, Akimoto $M$, Ishihara $Y$, et al. Evaluation of Dynamic Tumor-tracking Intensity-modulated Radiotherapy for Locally Advanced Pancreatic Cancer. Sci Rep. Springer US; 2018;8:17096.

22. Miften M, Olch A, Mihailidis D, Moran J, Pawlicki T, Molineu A, et al. Tolerance limits and methodologies for IMRT measurement-based verification QA: Recommendations of AAPM Task Group No. 218. Med Phys. 2018;45:e53-83.

23. Rubin P, Casarett GW. Clinical radiation pathology as applied to curative radiotherapy. Cancer. 1968;22:767-78.

24. Krishnan S, Chadha AS, Suh Y, Chen H-C, Rao A, Das P, et al. Focal Radiation Therapy Dose Escalation Improves Overall Survival in Locally Advanced Pancreatic Cancer Patients Receiving Induction Chemotherapy and Consolidative Chemoradiation. Int J Radiat Oncol Biol Phys. 2016;94:755-65. 
25. Nakamura A, Shibuya K, Matsuo Y, Nakamura M, Shiinoki T, Mizowaki T, et al. Analysis of dosimetric parameters associated with acute gastrointestinal toxicity and upper gastrointestinal bleeding in locally advanced pancreatic cancer patients treated with gemcitabine-based concurrent chemoradiotherapy. Int J Radiat Oncol Biol Phys. 2012;84:369-75.

26. Huang J, Robertson JM, Ye H, Margolis J, Nadeau L, Yan D. Dose-volume analysis of predictors for gastrointestinal toxicity after concurrent full-dose gemcitabine and radiotherapy for locally advanced pancreatic adenocarcinoma. Int J Radiat Oncol Biol Phys. 2012;83:1120-5.

27. Kelly P, Das P, Pinnix CC, Beddar S, Briere T, Pham M, et al. Duodenal toxicity after fractionated chemoradiation for unresectable pancreatic cancer. Int J Radiat Oncol Biol Phys. 2013;85:e143-9.

28. Liu X, Ren G, Li L, Xia T. Predictive dosimetric parameters for gastrointestinal toxicity with hypofractioned radiotherapy in pancreatic adenocarcinoma. Onco Targets Ther. 2016;9:2489-94.

29. Badiyan SN, Olsen JR, Lee AY, Yano M, Menias CO, Khwaja S, et al. Induction Chemotherapy Followed by Concurrent Full-dose Gemcitabine and Intensity-modulated Radiation Therapy for Borderline Resectable and Locally Advanced Pancreatic Adenocarcinoma. Am J Clin Oncol. 2016;39:1-7.

30. Krishnan S, Rana V, Janjan NA, Varadhachary GR, Abbruzzese JL, Das P, et al. Induction chemotherapy selects patients with locally advanced, unresectable pancreatic cancer for optimal benefit from consolidative chemoradiation therapy. Cancer. 2007;110:47-55.

31. Campbell WG, Jones BL, Schefter T, Goodman KA, Miften M. An evaluation of motion mitigation techniques for pancreatic SBRT. Radiother Oncol. 2017;124:168-73.

32. Lens E, Gurney-Champion OJ, Tekelenburg DR, van Kesteren Z, Parkes MJ, van Tienhoven G, et al. Abdominal organ motion during inhalation and exhalation breath-holds: pancreatic motion at different lung volumes compared. Radiother Oncol. 2016;121:268-75.

33. Nakamura M, Shibuya K, Shiinoki T, Matsuo Y, Nakamura A, Nakata M, et al. Positional reproducibility of pancreatic tumors under end-exhalation breath-hold conditions using a visual feedback technique. Int J Radiat Oncol Biol Phys. 2011;79:1565-71.

\section{Tables}

Table 1. Dose constraints for the planning study 
PTV boost*

$$
\mathrm{D}_{95 \%}=48 \text { Gy or } 45.6 \mathrm{~Gy}^{*}
$$

PTV

$$
\mathrm{D}_{98 \%} \geq 36 \text { Gy or } 34.2 \mathrm{~Gy}^{*}
$$

$$
\mathrm{D}_{\max }<56 \mathrm{~Gy}
$$

Stomach, duodenum, small bowel, and large bowel

$$
\mathrm{V}_{39 \mathrm{~Gy}} \leq 25 \mathrm{~cm}^{3}
$$

$$
\mathrm{V}_{42 \mathrm{~Gy}} \leq 5 \mathrm{~cm}^{3}
$$

$$
\mathrm{V}_{45 \mathrm{~Gy}} \leq 1 \mathrm{~cm}^{3}
$$

Stomach, duodenum, small bowel (PRV)

$$
\mathrm{V}_{36 \mathrm{~Gy}} \leq 45 \mathrm{~cm}^{3}
$$

$$
\mathrm{V}_{39 \mathrm{~Gy}} \leq 30 \mathrm{~cm}^{3}
$$

Spinal cord

$$
\mathrm{D}_{\max }<36 \mathrm{~Gy}
$$

Spinal cord (PRV)

$$
\mathrm{D}_{2 \mathrm{~cm} 3}<39 \mathrm{~Gy}
$$

Kidneys

Liver

$$
\mathrm{V}_{20 \mathrm{~Gy}}<30 \%
$$

$\mathrm{D}_{\text {mean }} \leq 30 \mathrm{~Gy}$

Abbreviations: $\mathrm{OARs}=$ organs at risk, $\mathrm{PRVs}=$ planning organs at risk volume, $\mathrm{V}_{\mathrm{xG}}=$ volume covered by the $\mathrm{x}$-Gy isodose line, $\mathrm{D}_{\max }=$ maximum dose volume, $\mathrm{D}_{2 \mathrm{~cm} 3}=$ dose covering $\geq 2 \mathrm{~cm}^{3}$ of the volume, $\mathrm{D}_{\text {mean }}=$ mean dose volume

*5\% reduction of the prescribed dose if PRVs dose constraints cannot be fulfilled.

Table 2. Dose volume indices of the target volumes 
Structures Indices DWA (Mean $\pm \mathrm{SD}) \quad$ co-VMAT (Mean $\pm \mathrm{SD}) \quad p$ value

\begin{tabular}{|c|c|c|c|c|}
\hline GTV & $\mathrm{D}_{98 \%}(\%)$ & $96.1 \pm 5.1$ & $96.9 \pm 5.4$ & 0.104 \\
& $\mathrm{D}_{95 \%}(\%)$ & $98.6 \pm 4.5$ & $99.2 \pm 4.9$ & 0.206 \\
& $\mathrm{D}_{50 \%}(\%)$ & $106.8 \pm 2.9$ & $106.6 \pm 2.8$ & 0.481 \\
& $\mathrm{D}_{2 \%}(\%)$ & $110.4 \pm 3.2$ & $109.8 \pm 3.0$ & 0.114 \\
\hline \multirow{2}{*}{ PTV } & $\mathrm{D}_{98 \%}(\%)$ & $79.0 \pm 4.3$ & $79.4 \pm 4.2$ & 0.133 \\
\hline & $\mathrm{D}_{95 \%}(\%)$ & $84.1 \pm 4.8$ & $84.6 \pm 4.8$ & 0.092 \\
\hline & $\mathrm{D}_{50 \%}(\%)$ & $106.3 \pm 1.9$ & $106.1 \pm 2.0$ & 0.174 \\
\hline & $\mathrm{D}_{2 \%}(\%)$ & $111.5 \pm 2.6$ & $110.5 \pm 2.4$ & 0.001 \\
\hline
\end{tabular}

Abbreviations: DWA $=$ dynamic wave arc, co-VMAT $=$ coplanar volumetric arc therapy, $\mathrm{SD}=$ standard deviation, GTV $=$ gross tumor volume, PTV = planning target volume, and $\mathrm{D}_{\mathrm{x} \%}=$ dose covered by the $\mathrm{x} \%$-isodose line

Table 3. Dose volume indices of the organs at risk other than gastrointestinal organs

\begin{tabular}{|c|c|c|c|c|c|}
\hline Structures & Indices & Dose constraints & $\begin{array}{c}\text { DWA } \\
(\text { Mean } \pm \text { SD) }\end{array}$ & $\begin{array}{c}\text { co-VMAT } \\
(\text { Mean } \pm \text { SD) }\end{array}$ & $p$ value \\
\hline \multirow[t]{2}{*}{ Rt kidney } & $\mathrm{V}_{20 \mathrm{~Gy}}(\%)$ & $<30 \%$ & $3.1 \pm 3.7$ & $4.8 \pm 5.4$ & 0.063 \\
\hline & Dmean (Gy) & Not defined & $20.8 \pm 5.5$ & $22.1 \pm 6.9$ & 0.096 \\
\hline \multirow[t]{2}{*}{ Lt kidney } & $\mathrm{V}_{20 \mathrm{~Gy}}(\%)$ & $<30 \%$ & $8.7 \pm 3.4$ & $7.8 \pm 4.2$ & 0.175 \\
\hline & $\mathrm{D}_{\text {mean }}(\mathrm{Gy})$ & Not defined & $23.3 \pm 3.8$ & $22.1 \pm 3.8$ & 0.057 \\
\hline Liver & $\mathrm{D}_{\text {mean }}(\mathrm{Gy})$ & $\leq 30 \mathrm{~Gy}$ & $6.9 \pm 3.5$ & $5.9 \pm 3.4$ & $<0.001$ \\
\hline Spinal cord & $\mathrm{D}_{\max }(\mathrm{Gy})$ & $<36$ Gy & $30.0 \pm 3.0$ & $29.0 \pm 3.4$ & 0.041 \\
\hline Spinal cord (PRV) & $\mathrm{D}_{2 \mathrm{~cm} 3}(\mathrm{~Gy})$ & $<39$ Gy & $31.7 \pm 2.8$ & $30.2 \pm 3.3$ & 0.011 \\
\hline
\end{tabular}

Abbreviations are the same as in Tables 1 and 2. 
Table 4. Proposed dose constraints for the duodenum in previous reports

\begin{tabular}{|c|c|c|c|c|c|}
\hline Author & $\begin{array}{c}\text { Prescribed } \\
\text { dose }\end{array}$ & Technique & Toxicity & $\begin{array}{l}\text { Proposed } \\
\text { constraints }\end{array}$ & $\begin{array}{l}\text { DVIs in this } \\
\text { study }\end{array}$ \\
\hline
\end{tabular}

\begin{tabular}{|c|c|c|c|c|c|}
\hline $\begin{array}{l}\text { Nakamura, } \\
\text { et al. [25] }\end{array}$ & $\begin{array}{c}54 \mathrm{~Gy} / 30 \\
\mathrm{fr}\end{array}$ & $3 \mathrm{D}-\mathrm{CRT}$ & $\begin{array}{c}\text { Acute } \\
\geq \text { Gr } 3 \\
\text { upper GI } \\
\text { bleeding }\end{array}$ & $\begin{array}{c}\mathrm{V}_{50 \mathrm{~Gy}} \square 33 \mathrm{~cm}^{3 *} \\
\left(\mathrm{BED}_{10}, 59 \mathrm{~Gy}_{10}\right)\end{array}$ & $\begin{array}{c}\mathrm{V}_{45 \mathrm{~Gy}}=0.2 \mathrm{~cm}^{3} \\
\left(0-1.3 \mathrm{~cm}^{3}\right)^{*} \\
\left(\mathrm{BED}_{10}, 59\right. \\
\left.\mathrm{Gy}_{10}\right)\end{array}$ \\
\hline $\begin{array}{l}\text { Huang, et } \\
\text { al. } \\
{[26]}\end{array}$ & $\begin{array}{c}36-42 \\
\mathrm{~Gy} / 15 \mathrm{fr}\end{array}$ & $\begin{array}{c}\text { 3D-CRT } \\
\text { IMRT }\end{array}$ & $\begin{array}{l}\text { Acute } \\
\geq \text { Gr } 3\end{array}$ & $\begin{array}{c}\mathrm{V}_{25 \mathrm{~Gy}} \mathrm{q} 45 \% \\
\left(\mathrm{BED}_{10}, 31-32\right. \\
\left.\mathrm{Gy}_{10}\right)\end{array}$ & $\begin{array}{c}\mathrm{V}_{25 \mathrm{~Gy}}=34 \% \\
(5-75 \%) \\
\left(\mathrm{BED}_{10}, 33\right. \\
\left.\mathrm{Gy}_{10}\right)\end{array}$ \\
\hline & $\begin{array}{c}30-38 \\
\text { Gy/15-19 } \\
\text { fr }\end{array}$ & & & $\begin{array}{c}\mathrm{V}_{35 \mathrm{~Gy}} \mathrm{q} 20 \% \\
\left(\mathrm{BED}_{10}, 43-45\right. \\
\left.\mathrm{Gy}_{10}\right)\end{array}$ & $\begin{array}{c}\mathrm{V}_{35 \mathrm{~Gy}}=12 \% \\
(0-39 \%) \\
\left(\mathrm{BED}_{10}=46\right. \\
\left.\mathrm{Gy}_{10}\right)\end{array}$ \\
\hline
\end{tabular}

\begin{tabular}{|c|c|c|c|c|c|}
\hline $\begin{array}{l}\text { Kelly, et } \\
\text { al. } \\
\text { [27] }\end{array}$ & $\begin{array}{c}50.4 \mathrm{~Gy} / 28 \\
\mathrm{fr} \\
57.5-75.4 \\
\mathrm{~Gy} / 28-39 \\
\mathrm{fr}\end{array}$ & $\begin{array}{c}\text { 3D-CRT } \\
\text { IMRT }\end{array}$ & $\begin{array}{l}\text { Acute } \\
\geq \text { Gr } 2\end{array}$ & $\begin{array}{c}\mathrm{V}_{\geq 55 \mathrm{~Gy}} \geq 1 \mathrm{~cm}^{3} \\
\left(\mathrm{BED}_{10}, 65-66\right. \\
\left.\mathrm{Gy}_{10}\right)\end{array}$ & $\begin{array}{c}\mathrm{V}_{\geq 48 G y}=0 \mathrm{~cm}^{3} \\
\left(0-0.04 \mathrm{~cm}^{3}\right) \\
\left(\mathrm{BED}_{10}, 63\right. \\
\left.\mathrm{Gy}_{10}\right)\end{array}$ \\
\hline
\end{tabular}

\begin{tabular}{|c|c|c|c|c|c|}
\hline $\begin{array}{c}\text { Liu, et al. } \\
\text { [28] }\end{array}$ & $\begin{array}{c}50 \mathrm{~Gy} / 15- \\
20 \mathrm{fr}\end{array}$ & IMRT & $\begin{array}{c}\text { Acute and } \\
\text { late } \\
\geq \text { Gr } 2\end{array}$ & $\begin{array}{c}\mathrm{V}_{45 \mathrm{~Gy}} \geq 0.5 \mathrm{~cm}^{3} \\
\left(\mathrm{BED}_{10}, 56-60\right. \\
\mathrm{Gy}_{10} ; \mathrm{BED}_{3}, 83- \\
\left.95 \mathrm{~Gy}_{3}\right)\end{array}$ & $\begin{array}{c}\mathrm{V}_{45 \mathrm{~Gy}}=0.2 \mathrm{~cm}^{3} \\
\left(0-0.7 \mathrm{~cm}^{3}\right) \\
\left(\mathrm{BED}_{10}, 59\right. \\
\mathrm{Gy}_{10} ; \mathrm{BED}_{3}, 93 \\
\left.\mathrm{~Gy}_{3}\right)\end{array}$ \\
\hline
\end{tabular}

* Volumes for the stomach plus duodenum are shown. 
Abbreviations: 3D-CRT $=$ three-dimensional conformal radiation therapy, DVIs $=$ dose volume indices, $\mathrm{DWA}=$ dynamic wave arc, $\mathrm{BED}=$ biologically effective dose, $\mathrm{GI}=$ gastrointestinal, $\mathrm{Gr}$ $=$ Grade, $\mathrm{fr}=$ fractions, $\mathrm{V}_{\mathrm{XG}}=$ volume receiving more than $\mathrm{X} \mathrm{Gy}, 3 \mathrm{D}-\mathrm{CRT}=$ three-dimensional conformal radiotherapy, IMRT $=$ intensity-modulated radiotherapy

\section{Figures}

(a)

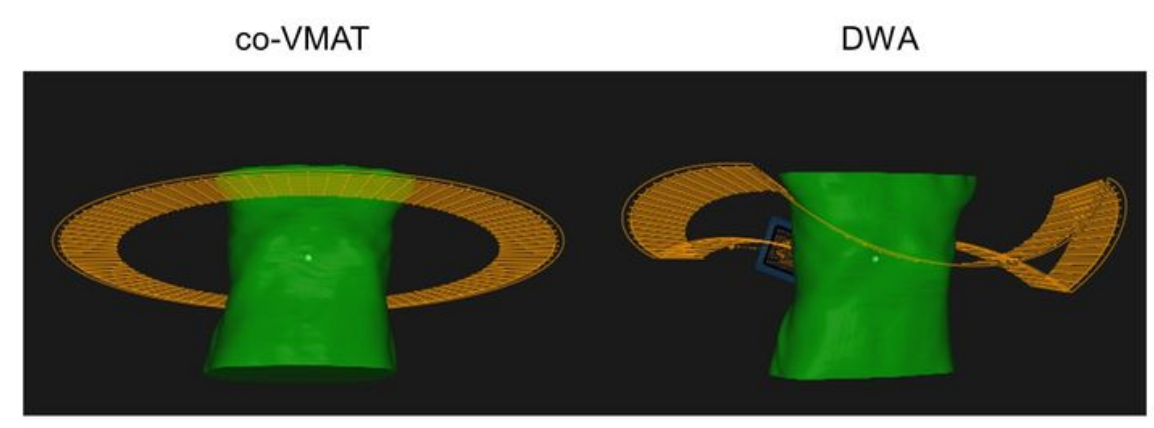

(b)

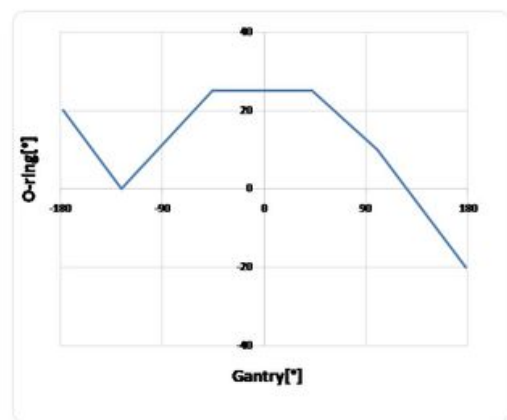

Figure 1

Diagrams presenting the gantry and O-ring rotation direction in addition to DWA delivery trajectory. (a) The 2D gantry-ring rotational positions. (b) The control points of the DWA delivery trajectory. 

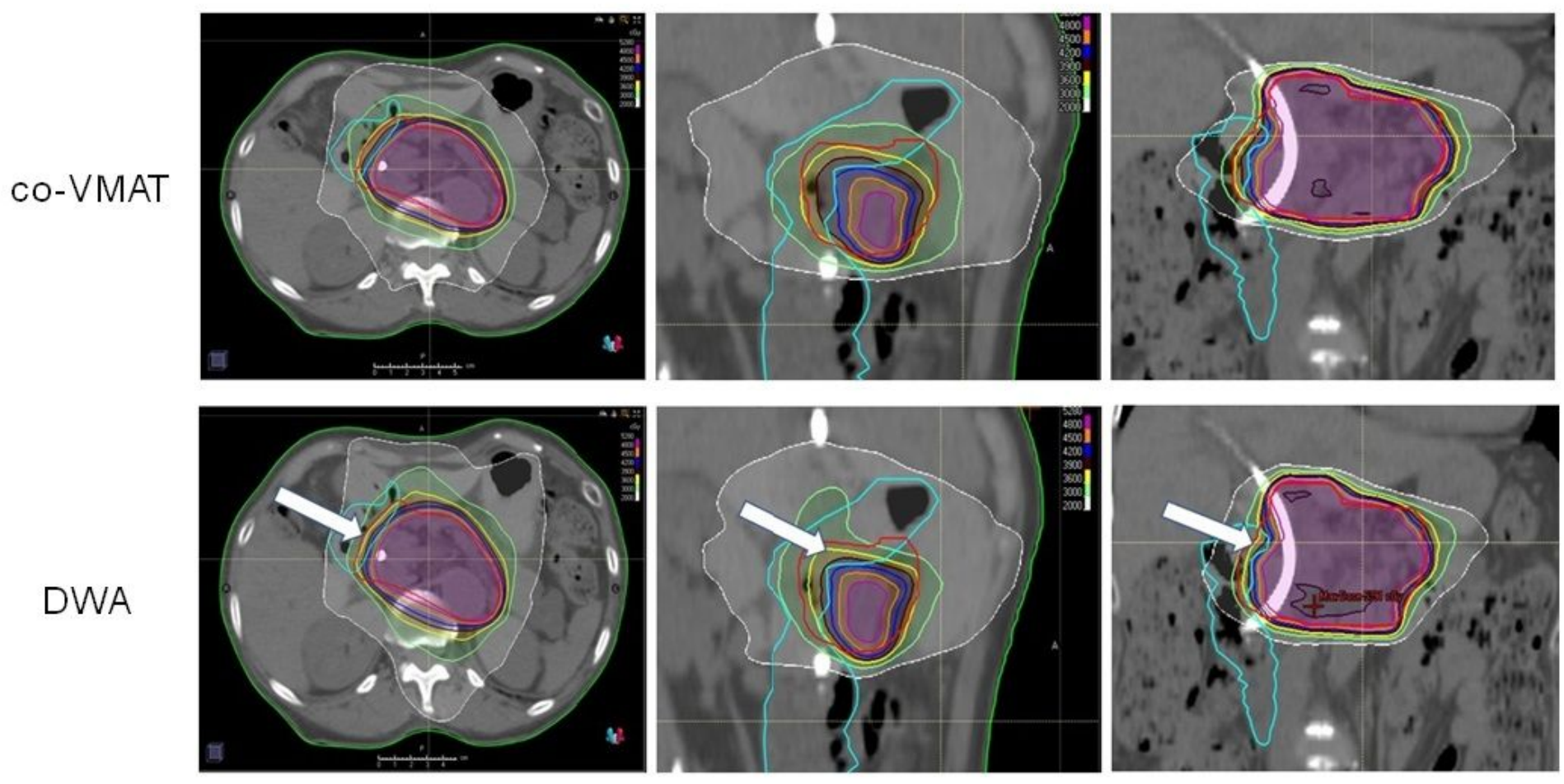

\section{Figure 2}

Dose distribution comparison between DWA and co-VMAT from a representative case. The PTV (red) is covered with an isodose line of 36 Gy (yellow). The white arrows indicate the duodenum (cyan) is spared in the DWA plan. 
(a)

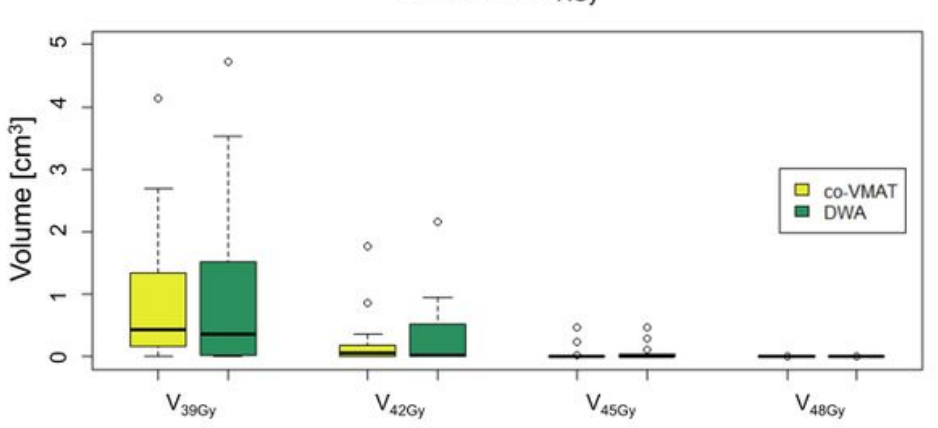

(c)

Small intestine $\mathrm{V}_{\mathrm{XG}}$

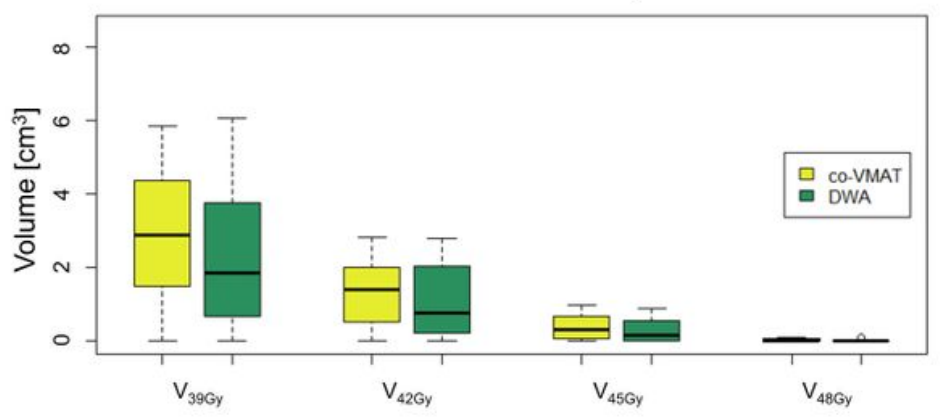

(b)

Duodenum $\mathrm{V}_{\mathrm{XGY}}$

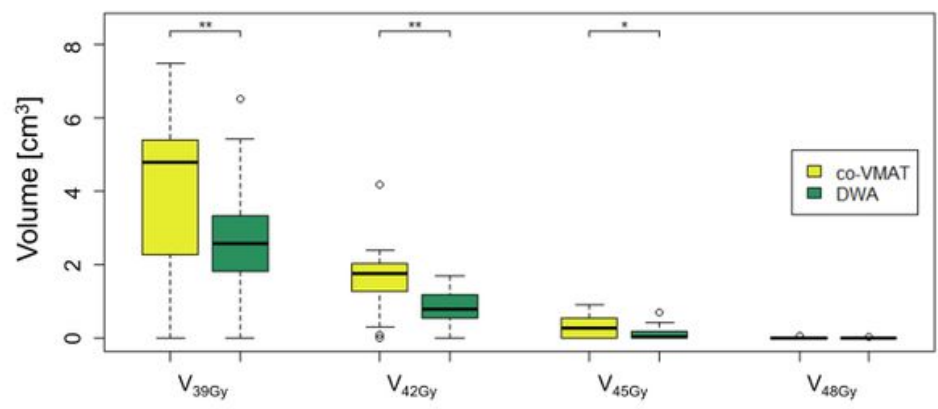

(d) Large intestine $\mathrm{V}_{\mathrm{XG}}$

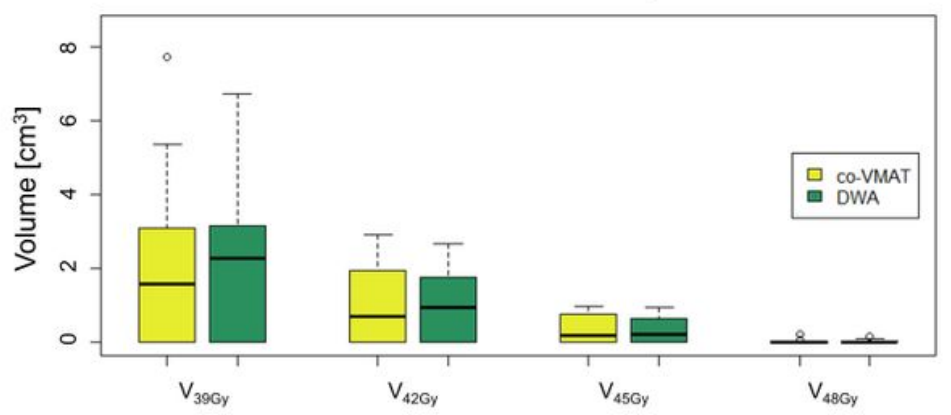

\section{Figure 3}

Box-and-whisker plots of the difference between DWA and co-VMAT in the gastrointestinal organs: (a) stomach, (b) duodenum, (c) small bowel, and (d) large bowel. The central box shows the values from the first to third quartiles. The horizontal line inside the box represents the median, and the vertical line indicates the range, except for outliers and far out values which are shown as 0 * indicates $p<0.05 ; * \star$ $\mathrm{p}<0.01$ 